\title{
Chronometric comparisons of imagery to action: Visualizing versus physically performing springboard dives
}

\author{
CATHERINE L. REED \\ University of Denver, Denver, Colorado
}

\begin{abstract}
Motor imagery research emphasizes similarities between the mental imagery of an action and its physical execution. In this study, temporal differences between motor imagery and its physical performance as a function of performer expertise, skill complexity, and spatial ability were investigated. Physical execution times for springboard dives were compared with visualized execution times. Results indicate that physical and visualized performance times were not identical: Their relation is a function of dive complexity and diver expertise, but not their interaction. Relative to physical time, visualization time increased with increased complexity, suggesting the involvement of capacity-limited working memory. A nonmonotonic relation was found for expertise: Unlike experts or novices, visualization time for intermediates was significantly slower than physical time. These temporal differences are most consistent with schematic differences in skill representation. Intermediates may be relatively slowed by greater amounts of nonautomatized knowledge, as compared with the automatized knowledge of experts or the sparse knowledge of novices.
\end{abstract}

Despite considerable research on the learning of simple motor acts (e.g., finger tapping), we know relatively little about the changes that occur in higher order representations of complex motor skills as expertise develops (Annett, 1995; Colley, 1989; Holding, 1989; Jeannerod, 1994; MacKay, 1989; Rosenbaum, Inhoff, \& Gordon, 1984; Schmidt, 1988). In the present study, the relation between the performance and the cognitive representations of complex motor skills was investigated. Motor imagery research has emphasized the similarity between the mental imagery of an action and its physical execution (Decety, 1996; Decety, Jeannerod, \& Preblanc, 1989; Decety \& Michel, 1989; Jeannerod, 1995, 1997). The present study focuses on how they might differ. Here, motor imagery was compared with the physical performance of springboard diving. The issue was whether the temporal relation between imagery and motor skill performance could reveal expertise-related differences in the content and structure of motor representations. Motor representations, or motor programs, have been defined as a set of movement components that are structured before a movement sequence begins (Keele, 1968). In addition, in this study, the

This paper is dedicated to my mentor, Daniel J. Weintraub. This study would never have left the diving board, so to speak, without the enthusiastic cooperation of four dedicated and knowledgeable diving coaches-Dick Kimball, Mike Lyden, Fred Woodruff, and Christine Seufert-and their diving teams. The author is grateful for the intellectual contributions of Eric Hughson, Dennis Egan, and Norman G. Vinson. Correspondence concerning this article should be addressed to C. L. Reed, Department of Psychology, University of Denver, 2155 S. Race St., Denver, CO 80208 (e-mail: creed@ du.edu). factors that influence motor imagery separately from motor representation were examined.

Several studies have suggested that physical and visualized motor skill performance share cognitive processes (Jeannerod, 1994; Requin, 1991). In hierarchical theories of motor control (MacKay, 1982, 1989; Shaffer, 1980, 1982 ) and theories of mental practice (Feltz \& Landers, 1983; Feltz, Landers, \& Becker, 1988), it is speculated that the two types of performance share the higher, cognitive levels of the hierarchy but differ in terms of the level at which performance output occurs. In experiments assessing practice and transfer effects for speech production, MacKay (1981) demonstrated that there are similarities between mental and physical practice and that mental execution times reflect internal processing. Internal speech execution times were faster than corresponding physical execution times, suggesting that internal action did not involve the activation of the movement's motor components.

The mental practice literature also supports a close relation between imagery and action (Feltz \& Landers, 1983; Feltz et al., 1988). Mental practice is the cognitive rehearsal of a motor skill that purportedly decreases errors in physical performance. Nonetheless, an athlete's expertise appears to influence the benefit that he or she receives from mental practice (Driskell, Copper, \& Moran, 1994; Richardson, 1967). Two current explanations for the positive influences of mental practice have been proposed. First, mental practice facilitates complex skills for which there is symbolic control of movements. It permits the rehearsal of a skill and an opportunity to code parts of the skill into meaningful cognitive units (Driskell et al., 1994). Minas (1978) suggested that mental practice reinforces 
the sequencing of movement components. Second, imagined activity produces impulses along the neural pathways associated with the action, thereby producing gains in performance (Hale, 1982; Kohl \& Roenker, 1983).

Neurological data provide converging evidence that imagined and executed actions share some neural substrates. Neuroimaging studies of motor imagery indicate metabolic activity in brain areas associated with both motor preparation and performance (Decety, 1996; Decety \& Ingvar, 1990; Decety, Kawashima, Gulyas, \& Roland, 1992; Roland, Larsen, Lassen, \& Skinhoj, 1980). Studies of neurologically impaired patients also have demonstrated a concurrent involvement of cortical areas involved in motor imagery and performance. For hemiplegic patients, motor imagery and movement times for the paralyzed limb were mentally slower than those for the normal limb (Decety \& Boisson, 1990). Similarly, visualization times reflected the impaired or slowed physical performance of patients with brain damage or neurodegenerative diseases (Dominey, Decety, Broussolle, Chazot, \& Jeannerod, 1995; Sirigu et al., 1996).

Likewise, autonomic responses during motor imagery parallel those of physical movement (Decety, 1996). Motor imagery and physical execution appear to activate similar muscles and motor programs (Kohl \& Roenker, 1983). Studies using EMG recordings show that imagined activity produces low-level motor effects that correspond to patterns of action (Hale, 1982; Harris \& Robinson, 1986; Jacobson, 1932; McGuigan, 1970; Ulich, 1967). Furthermore, the similarity between the timing of motor imagery and that of physical performance implies the activation of similar cognitive structures (e.g., Decety \& Michel, 1989; Landauer, 1962). When participants were asked to move their hand into the orientation of a hand stimulus or to mentally simulate that action, mental simulation times mimicked movement times (Parsons, 1994).

Despite these similarities, the relation between physical and visualized movements is not necessarily simple. For example, task difficulty has a greater influence on visualized times than on physical times (Decety, 1991; Decety \& Jeannerod, 1996; Decety et al., 1989). In addition, muscular responses during mental imagery differ as a function of expertise. During skill imagery, advanced performers produced increased EMG activity in skill-specific muscles, as compared with beginning performers (Harris \& Robinson, 1986). These findings suggest that the temporal relation between visualized and physical performances may not be one to one and that task difficulty and expertise level may influence it.

In the present study, the cognitive organization of a complex motor skill was investigated by comparing visualized and physical performance times. Springboard diving was selected as a model real-world motor skill because diving from a board requires both a concrete cognitive representation of one's orientation in space and a cognitive set of sequentially organized movements. Each diver's visualized dive could be compared against his or her physical dive. Comparisons of these corre- sponding times may provide evidence for representational differences that depend on diver expertise and motor skill complexity. Furthermore, performance times can be sampled periodically throughout the dive. Unlike a single overall dive time measure, waypoint measures allow an assessment of the time course of mental processing.

Research on the relation between visualized and physical performance times has suggested that visualized performance activates the same motor programs as the physical performance (Jeannerod, 1994; Requin, 1991). If this is true, the present results should show that visualized performance times follow physical performance times at the same rate. However, visualized performance may be faster because it is not connected to motor output (MacKay, 1982).

Expertise is thought to modify the cognitive representations of complex motor skills. Thus, it may affect performance times in several ways. If expertise has a fine-tuning effect, in that the same motor program is constructed the same way every time, greater expertise may decrease the discrepancy between visualized and physical performance times and reduce the variability of performance (Jannson, 1983).

Alternatively, expertise may produce timing differences consistent with changes in schematic skill knowledge. People demonstrate nonlinear changes in their understanding and performance of both cognitive and motor skills as they improve (Anderson, 1983; Rosenbaum, 1991). These changes in schematic skill knowledge may be reflected in a nonmonotonic discrepancy between visualized and physical performance times across expert, intermediate, and novice expertise levels. For example, expert divers have practiced and refined their dives to the point of automaticity. As a result, they should produce visualized times that are closely related to physical performance times. In contrast, intermediate divers have extensive knowledge about how to perform dives, but their performance is not fully automatic. Thus, the time to put the components of the dive together for visualized performance may produce relatively longer visualized than physical performance times. The slowing of the mental process may be less evident for physical performances to the extent that the physical properties of the diving board, the body, and gravity constrain the duration of the dive. Last, novice divers have less schematic knowledge about how to perform the dives. With fewer cognitive options to coordinate for dive performance, their visualization times may be relatively faster.

The rotational complexity of the skill may influence the visualized/physical temporal relation (Decety, 1991; Decety \& Jeannerod, 1996). The dives selected for this study represented three levels of rotational complexity and task difficulty: no body rotation, rotation around one body axis, and simultaneous rotation around two body axes. Rotational complexity provides a reasonable operational definition of task difficulty. Even though the visualized body and the physical body may be subject to similar biomechanical constraints (Parsons, 1994), additional 
transformations of visualized dives may require more time than their physical counterparts (Just \& Carpenter, 1985). If visualized performance occurs in a limited-capacity processing system (i.e., working memory) for which additional visualized spatial rotations require extra time to execute, visualized performance time should increase with rotational complexity.

Generalized, nonspecific spatial ability may also be related to the ability to visualize motor skills. Individual differences in visual imagery and on spatial ability tests appear to be related to the capacity of spatial working memory (Just \& Carpenter, 1985; Kosslyn, Brunn, Cave, \& Wallach, 1984; Kosslyn \& Schwartz, 1977). People who have high scores on spatial ability tests may have greater spatial working memory capacities, allowing them to visualize more complex image transformations. In this task, effects of expertise may simply reflect this spatial ability: The ability to visualize body transformations accurately and easily may decrease the difference between visualized and physical performance times. To assess this hypothesis, participants were grouped on the basis of their combined scores on two standardized, spatial ability tests that required the mental reorientation of nonbody objects.

To investigate whether a cued memory representation influences visualized performance times, the effects of viewing a videotaped dive prior to visualization were examined. Dive previewing provided temporal and skillspecific visual information that could prime the dive's representation. Such cues could decrease the disparity between visualized and physical times, especially for less skilled divers. The priming of an individual's own body representation and motor skill components could enhance the effect by activating specific action representations.

\section{METHOD}

\section{Participants}

Twenty-six springboard divers (9 male, 17 female; $13-22$ years old) volunteered from the University of Michigan, Eastern Michigan University, Lords of the Boards, and Brighton diving teams. Informed consent was obtained, and the divers' rights as participants were protected. Diving experience ranged from 2 to 18 years. Despite efforts to select divers who could complete the full experiment, 4 divers were excluded from analyses because they could not perform the most difficult dive. Divers were ranked according to expertise as defined by two coaches familiar with the divers and by judges' scores received during competition. The two coaches had $100 \%$ reliability in their rankings. Given the existing skill levels of the particular divers tested, the number of divers making up each group was not uniform. The expert group consisted of 7 divers ( 3 male, 4 female), the intermediate group had 8 divers ( 3 male, 5 female), and the novice group had 7 divers ( 3 male, 4 female). All of the divers in the expert group competed in the U.S. Olympic Trials, and more than half later competed in the Olympics. The divers in the intermediate group competed in collegiate-level, but not in national-level, competitions. The divers in the novice group competed at regional-level competitions.

\section{Apparatus}

Physical dives were performed on 3-m indoor diving boards. They were recorded on videotape from a $90^{\circ}$ angle via a video camera located on the pool deck. The dives were captured from initiation to water entry. Physical performance times were recorded on the video camera's timer to the nearest $0.01 \mathrm{sec}$ and were recalibrated to real time.

Visualized performance data were collected using a custommade timer. A pair of response boxes was connected to a central timing unit that recorded time with millisecond accuracy.

\section{Stimuli}

Three dives were selected using two criteria: (1) All the divers could perform the same dives, and (2) each dive represented a different rotational complexity (see the Appendix). Dive numbers are from the AAU Diving Handbook (1985). The front dive (Dive 101: forward dive, straight position) traces out a simple arc to a headfirst water entry. The front somersault (Dive 10: forward dive, two-andone-half somersault, pike position) adds rotation about the body's lateral (side-to-side) axis, body bent at the hips. The back twister (Dive 5,231: back dive, one-and-one-half somersault, one-and-onehalf twists, free position) requires rotations of the unbent body about both the lateral axis and the longitudinal (head-to-toe) axis and begins with the diver facing backward, heels extending past the end of the springboard. Videotapes of these dives were acquired in the first session and were used as stimuli in the second session.

The Guilford-Zimmerman Aptitude Survey Test (Part VI: Spatial Visualization; Guilford \& Zimmerman, 1947) and the Thurstone Flags Test (Thurstone \& Jeffreys, 1956) were used to assess generalized spatial ability and mental manipulation skills. In the Spatial Visualization test, the participants rotated clocks mentally to match targets. In the Flags Test, the participants rotated flags mentally to match targets. These tests were selected because they required the mental manipulation of nonbody objects and had normalized scoring procedures.

\section{Procedure}

The divers were tested during their regular practice sessions at their pools and in rooms separate from the pool area. They were told that the purpose of the study was to better understand how mental dive visualization was related to dive performance.

First, the divers executed nine dives on the diving board, with the three required dives performed three times each. The divers were given cards with the dives listed in the specified order of execution, which was rotated over divers. Each dive was performed once before repeating the order. The divers lined up at the diving board and performed their dives sequentially. The dives were recorded on videotape. This part took approximately $20 \mathrm{~min}$.

After completing the physical dive portion of the study, the divers timed their visualizations of each dive. The time between physical performance and visualization was approximately $20 \mathrm{~min}$. The divers were told to imagine themselves diving as if they were actually executing the dive or to mentally simulate their physical dive as closely as possible. In addition to timing visualizations of complete dives, the divers were asked to time various subcomponents of the dives, or waypoints. Each waypoint started at the beginning of the dive at $0 \mathrm{sec}$. Successive waypoints included an increasingly greater part of the complete dive. W1 started at the initiation of the first dive movement and ended at the beginning of the hurdle. W2 included the hurdle and ended when the diver departed the springboard for good. W3 ended at the completion of the aerial maneuver when the diver commenced recovery. W4 ended at water entry. For example, the timelines of the forward dive and the forward somersault dive were the following: Start [approach] W1 [hurdle] W2 [aerobatics] W3 [recovery] W4 [water entry]. The back twister began at the end of the board and had only three waypoints: Start [hurdle] W2 [aerobatics] W3 [recovery] W4 [water entry]. All the divers reported that they routinely visualized themselves performing dives and that they were highly familiar with the specified waypoints.

Before beginning visualization trials, the divers were questioned in order to ensure that they understood the instructions. With the dis- 
play unit hidden, the divers held one timer box in each hand while visualizing a dive. They pressed the start button with their right thumbs and the stop button with their left thumbs. For each trial, the divers were told which dive to visualize and at which waypoint to stop the timer. A single waypoint time was measured per visualization trial. Upon initiation of the first visualized body movement, the divers pressed the timer's start button. When their mental representations arrived at the specified waypoint, they pressed the stop button. Regardless of the waypoint at which they stopped the timer, the divers were instructed to always visualize the complete dive from start to finish. Thus, for all the waypoints measured, the divers stopped the timer, but never the visualization. Complete dives were visualized to make the cognitive execution of the dives as uniform as possible across trials, sessions, and divers. Although the buttonpress may have disrupted normal cognitive processing, it was constant across all conditions.

The divers visualized the dives in the same order as they had physically performed them. The only difference was that after they visualized the entire dive, they then visualized successive waypoints within the dive. After completing each of the three dives once, the sequence was repeated until the divers visualized each waypoint for each dive three times. The visualization portion of the study took approximately $20 \mathrm{~min}$ for each diver. The entire first session took approximately $1 \mathrm{~h}$ for each diver to complete.

In a second session, the divers participated in a videotapepreviewing task and completed two pencil-and-paper spatial ability tests. This session was conducted in the same rooms as the first session. For the previewing task, the divers first visualized nine dives in the order in which they had physically performed them. The divers visualized each dive from start to water entry, following the same procedure as that used in Session 1. Keeping the same order, they then viewed individual dives from the videotape of themselves performing the dives in the first session (preview self). The corner of the screen on which the timer was displayed was covered. Last, they viewed the videotape of another diver of the same gender performing the same dives (preview other). For both conditions, the divers viewed a videotape of a single dive and immediately thereafter timed a visualization of that dive. The dives were viewed and visualized three times each.

Finally, spatial ability tests were administered according to the test instructions. The tests required the divers to compare two nonbody objects (i.e., clocks and flags) and to determine whether an item matched a target. The second session took approximately 45 min to complete.

\section{RESULTS AND DISCUSSION}

Physical performance waypoint times were extracted from the videotaped physical performances, using the frame-by-frame advancement feature on a videotape recorder. Mean physical and visualized performance times were computed for each waypoint for each participant. Dive segment times were then computed from the waypoint measures. Dive segments were time intervals defined by successive waypoints within a dive, with each segment representing a unique part of the dive. Unlike waypoints, no part of any segment was shared by other segments. The approach segment included the time from the start to stepping on the end of the board (i.e., the end of W1). The hurdle segment included the time starting with the bouncing on the end of the board to the final liftoff (i.e., the end of W2 minus the approach segment). The aerobatics segment included the required aerial maneuvers (i.e., the end of $\mathrm{W} 3$ minus the hurdle segment).
Last, the recovery segment included the preparation for water entry to water entry (i.e., W4 minus the aerobatics segment). In sum, each segment excluded the time of the previous segment.

To illustrate the differences between types of performance, mean waypoint data for physical performance time was plotted against visualized performance time for each dive and participant. These effects are illustrated in Figure 1. A regression line was fit to this data, and the slope was calculated. A group $\times$ dive analysis of variance (ANOVA) was conducted using slope data. Significant effects were found for group $\left[F(2,19)=5.40, M S_{\mathrm{e}}=0.19, p<.02\right]$ and dive $\left[F(2,38)=13.15, M S_{\mathrm{e}}=0.02, p<.0001\right]$. There was no group $\times$ dive interaction $\left[F(4,38)<1\right.$, n.s.]. ${ }^{1}$

Although there was a systematic relation between performance types, physical times were not identical to visualized times. Differences between visualized and physical performance times increased with each additional body-axis rotation. In addition, expertise level had an overall effect on the dive time relation that was independent of the particular dive. Expert divers visualized their dives closest to their performance times. Intermediate divers visualized their dives slower than their performance times. Novice divers visualized their dives faster than performance times.

To examine differences between the two types of performance times at various points within the dive, a difference measure was calculated using dive segments. Mean physical performance time was subtracted from mean visualized performance time. Positive differences indicated that visualization times were faster than physical times. A linear regression analysis with dummy variables was conducted so that interpretations could be generalized across the dives; it permitted unbalanced designs and had a test for the effects of nonindependent data. ${ }^{2}$ In addition, the analysis tested the precise questions of interest: Was there an effect of dive when group was held constant? Was there an effect of group when dive was held constant? Was there an interaction between group and dive?

For the analyses below, a regression with dummy variables of group and dive was conducted using the segment difference scores. An $F$ test for group, with dive held constant, revealed a significant effect $[F(6,233)=4.67, p<$ $.0002]$. Difference scores for the intermediate group were largest, as well as negative. In contrast, expert and novice groups had positive differences. These results are more consistent with expertise differences in motor skill representations than with a fine-tuning explanation for which expertise produces a systematic reduction in the performance time relation.

Next, differences for individual segments within the dives were examined. Two segments were of particular interest: the approach and the aerobatics segments. The approach segment may reflect the assembly of the motor components to be executed. The aerobatics segment may reflect differences in the selection of components. Analyses of the different components revealed a group effect for the approach segment $[F(6,233)=2.71, p<.02]$, but 

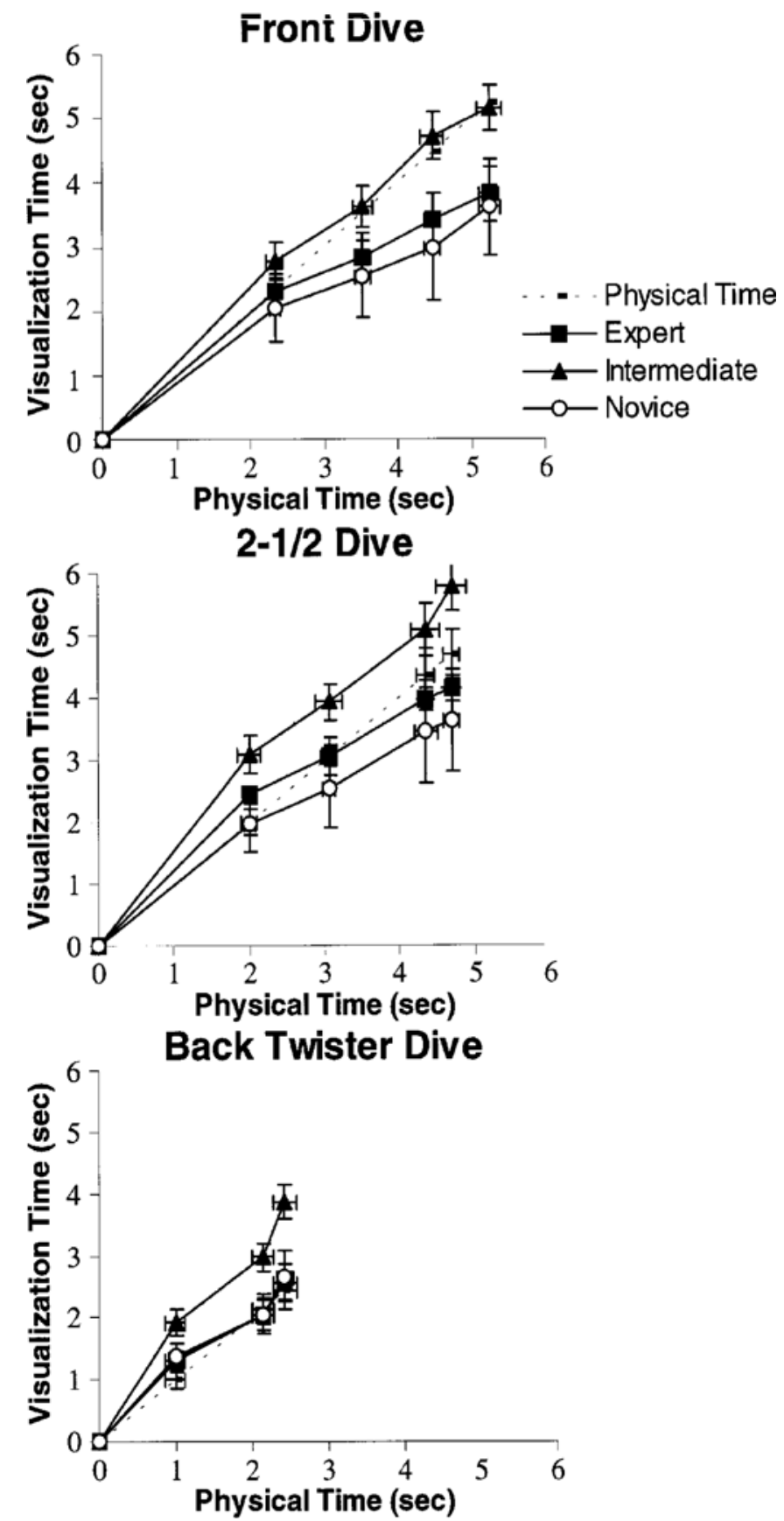

Figure 1. Mean visualized waypoint times (in seconds) plotted against corresponding physical waypoint times (in seconds) as a function of group (expertise level: expert, intermediate, and novice) and dive (rotational complexity: front dive, front somersault, and back twister). The $45^{\circ}$ line indicates equal performance times. The vertical error bars indicate the standard error for each visualized performance time waypoint. As a point of comparison, the horizontal error bar indicates the standard error for each physical performance time waypoint.

not for the aerobatics component $[F(6,233)=1.43$, n.s. $]$. These analyses confirm the value of the individual segment analyses and support the idea that much of the cognitive activity and organization of dive execution occurs early in preparation stages.
By using segment difference scores to investigate effects of rotational complexity, a significant effect was found for dive, with group held constant $[F(6,233)=3.14$, $p<.006]$. Visualization times were slower relative to physical performance times as the rotational complexity 
of the dive increased. This result is particularly striking for the most complex dive, the back twister, which requires simultaneous rotation around both vertical and horizontal axes. This pattern occurred for all expertise levels, with no group $\times$ dive interaction $[F(4,233)<1$, n.s. $]$. The dive and group effects appear to arise from different mechanisms. The dive effect may be related to mental simulation processes, rather than to expertise differences in mental representations.

Within-subjects performance variability was computed for each participant and condition by squaring the difference between the trial time for the waypoint and the overall waypoint mean for each dive (Table 1). Physical performance variability was analyzed using a regression with dummy variables of group and dive. No significant $F$ tests were found for the effect of group with dive held constant $[F(6,188)=1.20$, n.s. $]$, for the effect of dive with group held constant $[F(6,188)=1.61$, n.s. $]$, or for the group $X$ dive interaction $[F(4,188)=1.20$, n.s.]. Similar analyses for visualized performance variability did not produce significant $F$ tests for the effects of group when dive was held constant $[F(6,188)<1.0$, n.s.] or dive when group was held constant $[F(6,188)<1$, n.s.] or for the group $\times$ dive interaction $[F(4,188)<1$, n.s.]. Within a single diving session, expert divers were not more consistent in the timing of their dives, and difficult dives were not more variable in their execution times. The predicted differences in variability across skill levels may be detected only across performance sessions, rather than across dives within a particular session.

A nonmotor factor that may drive the physical-visualized performance time relation is the ability to mentally manipulate objects. Scores from the two spatial ability tests were summed to provide a more reliable measure. On the basis of this measure, the participants were grouped into high, intermediate, and low spatial ability groups. ${ }^{3} \mathrm{~A}$ spatial ability group $\times$ dive ANOVA was conducted using slope data. A significant effect was found for dive $\left[F(2,38)=16.87, M S_{\mathrm{e}}=0.019, p<.0001\right)$, but not for group $\left[F(2,19)<1, M S_{\mathrm{e}}=0.27\right.$, n.s. $)$, nor was there a significant group $\times$ dive interaction $\left[F(4,38)=2.46, M S_{\mathrm{e}}=\right.$ 0.019 , n.s.]. In addition, regression analyses were conducted with dummy variables of spatial ability and dive for segment-difference measures. No significant effects were found for spatial ability when dive was held constant $[F(6,233)<1$, n.s. $]$. An effect of dive with spatial ability held constant was found $[F(6,233)=3.06, p<.007]$. More time may be required to execute additional mental transformations.

To determine whether the high spatial ability group was able to visualize mental transformations faster than were the other two groups, a regression was conducted with the dummy variables of spatial ability and dive for visualized segment times. No significant effect was found for spatial ability with dive held constant $[F(6,233)<1$, n.s.], nor was there a significant interaction $[F(4,233)<1$, n.s.]. The ability to mentally manipulate objects did not influence the relation between physical and visualized performance times or even visualization times alone.

To investigate whether visualized performance times could be cued by prior viewing of a videotaped dive, an expertise group $\times$ dive $\times$ condition (no preview, viewing oneself, viewing another) ANOVA was performed on mean data for visualized dives from the second testing session (Table 2). A main effect of group replicated the nonmonotonic expertise effect $\left[F(2,19)=4.28, M S_{\mathrm{e}}=\right.$ $10.66, p<.03]$ : The intermediate group produced longer visualization times than did the expert and novice groups. A main effect of dive reflected the faster visualization (and faster physical execution) of the back twister dive $\left[F(2,38)=85.26, M S_{\mathrm{e}}=0.35, p<.0001\right]$. A main effect for condition indicated a general speeding effect of video viewing $\left[F(2,38)=4.15, M S_{\mathrm{e}}=1.93, p<.03\right]$. Videotape cuing may remind participants of the speed and simultaneity of the rotations. However, no significant differences were found between viewing oneself or viewing another performing the dive. The group $\times$ dive interaction indicated that novices produced similar visualization times for all three dives but that the expert and intermediate groups produced faster visualization times for the back twister than for the other two dives $[F(4,38)=3.61$, $\left.M S_{\mathrm{e}}=0.35, p<.02\right]$. There was no significant group $\times$ condition interaction $[F(4,38)<1$, n.s.], dive $\times$ condition interaction $\left[F(4,76)=1.89, M S_{\mathrm{e}}=0.09\right.$, n.s.], or threeway interaction $[F(8,76)<1$, n.s.]. Previewing did not have differential priming effects for the expertise groups.

\section{GENERAL DISCUSSION}

For the past decade, researchers have presented evidence to support the hypothesis that motor imagery mirrors motor performance. They have argued that the same

Table 1

Within-Subjects Variability for Physical and Visualized Performance Times (in Seconds) for Waypoint Data

\begin{tabular}{lcccc}
\hline \multirow{2}{*}{ Group } & Performance Type & Front & Front Somersault & Back Twister \\
\cline { 3 - 5 } Expert & physical & 0.031 & 0.030 & 0.004 \\
& visualized & 0.114 & 0.118 & 0.099 \\
Intermediate & physical & 0.032 & 0.016 & 0.013 \\
& visualized & 0.178 & 0.083 & 0.179 \\
Novice & physical & 0.018 & 0.026 & 0.032 \\
& visualized & 0.107 & 0.123 & 0.077 \\
\hline
\end{tabular}


Table 2

Mean Visualization Performance Times (in Seconds) for Complete Dives by Group (Expertise Level) and Video Preview Condition

\begin{tabular}{llccc}
\hline & & \multicolumn{3}{c}{ Visualization Condition } \\
\cline { 3 - 5 } Group & \multicolumn{1}{c}{ Dive } & No Preview & Preview Self & $\begin{array}{c}\text { Preview } \\
\text { Other }\end{array}$ \\
\hline Expert & front & 3.77 & 3.58 & 3.56 \\
& front somersault & 4.09 & 3.82 & 3.66 \\
\multirow{4}{*}{ Intermediate } & back twister & 2.51 & 2.23 & 2.47 \\
& front & 5.22 & 4.58 & 4.87 \\
& front somersault & 5.63 & 4.69 & 5.21 \\
\multirow{3}{*}{ Novice } & back twister & 3.73 & 3.25 & 3.57 \\
& front & 3.82 & 2.53 & 2.89 \\
& front somersault & 3.96 & 2.68 & 2.80 \\
& back twister & 2.96 & 2.06 & 2.17 \\
\hline
\end{tabular}

mental representations are tapped during the perception, imaging, memory, and performance of an action (e.g., Decety, 1996). The present study has demonstrated that this classic conclusion may be overly simplistic. It examined springboard diving, a complex motor skill, to compare the temporal aspects of physically complex motor skill performance relative to motor skill imagery. Skill complexity and diver expertise were manipulated.

The results showed that physical and visualized performance times were not identical: Their relation was a function of rotational complexity and diver expertise, but not their interaction. Overall, visualization time increased relative to physical time with increased skill complexity. A nonmonotonic relation was found for expertise level: Unlike experts or novices, the visualization time for intermediates was significantly slower than physical performance time. Thus, the temporal aspects of motor imagery were not simply a direct, or even a uniformly shifted, version of the temporal aspects of motor production. These results demonstrated that there are important mental processing differences between action performance and action visualization. Furthermore, action representation and imagery cannot be understood without taking into account the processing constraints imposed by the visual and working memory systems.

Overall, the results support prior work. Visualized performance times followed physical performance times at similar rates, and the speed of visualized performance was generally faster than that of physical performance, because it was not connected to motor output (MacKay, 1982). Distinctions between imagery and action are consistent with neuroimaging studies that have found overlapping but different neurophysiological substrates: The premotor cortex is active during motor imaging, whereas the premotor cortex plus the primary and later motor cortices are active during action performance (Jeannerod \& Frak, 1999).

Extending previous findings, the present study demonstrated that chronometric differences between imagery and action are influenced by expertise. The hypothesis of a fine-tuning mechanism in which the temporal discrepancy between visualized and physical performances decreased with expertise was not supported. Instead, the re- sults were more consistent with changes in schematic skill knowledge. One explanation for a nonmonotonic performance time relation is that expertise influences the cognitive organization of skill (Anderson, 1983; KarmiloffSmith, 1990, 1992). For example, intermediate divers have considerable knowledge about how to perform their dives, but their performance is not fully automatic. Thus, the time to assemble dive components for visualized performance may be relatively longer than that of expert divers, for whom component assembly is automatized. Novice divers produce relatively faster visualization times than do intermediate divers because they have less schematic knowledge about the dives to assemble for dive performance. Supporting this idea, intermediate-level athletes benefit the most from mental practice (Minas, 1978). Something different appears to be going on for performers at this level, whether mental practice reinforces the sequence or the appropriate selection of movement components. The relative slowing or speeding of the visualized performance of divers at different expertise levels is not found for physical performances, because the physical properties of the diving board, the body, and gravity constrain the duration of physical performance.

Although complete dive durations address overall expertise differences, they do not reveal when in the process the time differences among groups emerge. In these data, the approach segment for both physical and visualized performance produced the greatest expertise-level timing differences. Experts spent proportionately more physical time on the approach segment, and intermediates spent proportionately more visualization time on it. The fact that the changes occurred early in processing time implies that movement preparation may be critical for skilled execution of a complex action. Skill-level differences may be related to the assembly of the complex motor representation. Performers may use this time for the selection and sequencing of movement components. For performers and instructors, the implication is that performers should not rush the initial preparatory components of a complex motor skill. In sum, the cognitive processes and representations used to perform complex motor skills appear to change with skill development, and initial preparation segments of skill execution may reflect this process. 
Skill or rotation complexity also influences the temporal correspondences between physical and visualized performances. Generally, visualized time increased relative to physical time as rotational complexity increased. For example, the back twister requires the simultaneous rotation of the body in three dimensions. Although its actual timing is performed much faster than the other dives, it is visualized at a slower-than-real-time rate. Visualization data, as well as spontaneous diver reports, suggest that divers either rotated objects sequentially around each axis or decreased the rate of mental rotation for complex rotations. Because this finding held for all levels of expertise, visualized performance may not be based purely in physical experience.

Thus, properties of the imagery system also appear to influence the temporal relation between imagery and action. Visualized performance may occur in a limitedcapacity processing system that affects the speed and number of image transformations that can occur simultaneously (Kosslyn \& Schwartz, 1977). Additional or more difficult transformations of objects in general take more time to complete (Just \& Carpenter, 1985; Kosslyn $\&$ Koenig, 1992). In terms of the present results, the complex rotations required by the back twister appeared to exceed the divers' ability to imagine them simultaneously. The medium in which motor imagery occurs appears to influence temporal relations independently of motor representation processes. Its limitations seem to influence the rate of visualized performance (Kosslyn et al., 1984). Thus, the present study was able to separate central representational effects from the medium in which they occurred.

In contrast to expertise and complexity, generalized spatial ability, or the ability to manipulate nonbody objects through complex mental rotations, did not influence the imagery-action relation. Although mental transformations are required for imagining dives, there was no difference between divers with high and low spatial abilities for dive visualization rates or performance time relations. Nonetheless, whether experienced divers are better at mentally rotating the body in space is an open question. Several studies have provided evidence that the human body is represented and processed differently from other objects (e.g., Ogden, 1985; Parsons, 1987a, 1987b, 1994; Reed \& Farah, 1995; Shiffrar \& Freyd, 1993). Spatial ability tests that require the manipulation of body parts (e.g., the hand, foot, and body tests used by Parsons, 1987a, $1987 b$ ) might produce different results.

In addition, neither expertise nor skill complexity affected performance consistency, a hallmark of skilled performers. Two factors may have influenced the findings. First, the effects of expertise may have been reduced by the selection of dives and divers. Dives were selected that all the participant divers could perform. Second, expertiserelated consistency may be observed reliably only across sessions, rather than within sessions, as measured here.

Finally, the priming of the dive representation by viewing dive videotapes generally speeded visualization times, but not as a function of expertise or type of priming. No difference was found between using primes of one's own or another's physical performance. Cuing effects did not depend on the external activation of the performer's own body representation. This result is consistent with neurological research indicating a neural equivalence for actions performed by self and others (e.g., Rizzolatti, Fadiga, Gallese, \& Fogassi, 1996). In the monkey premotor cortex, mirror neurons fire when the monkey performs an action and when he observes a similar action performed by someone else. Thus, correspondences exist between observation of an action at both neural and behavioral levels, whether the action is performed by the self or another.

Some caution must be taken with respect to the generalizability of the present findings. The sample sizes and the number of observations per participant were relatively small. The nonmonotonicity of the expert and novice groups, relative to the intermediate group, may have been an aberration. However, some data provide support for the present conclusions. First, the visualization times of the second testing session replicated the visualization times of the first testing session, in that the intermediate group's times were longer than those of the expert and novice groups. Second, a different set of intermediate and novice divers was tested at a later time. Again, the intermediate group's visualization times were slower than those of the novice group. Expert divers were not available to determine whether similarities between expert and novice divers were maintained.

This study points to issues that current theories of motor skill acquisition should address. These theories account for performance and representational changes that occur during simple motor skill acquisition (Ivry, 1994; Schmidt, 1975, 1988). Error feedback and strengthening of movement components acquired through extensive practice produce linear refinements in the representation and corresponding improvements in performance. However, these theories do not account for perturbations in the continuity of representational change, especially after considerable skill has been acquired. The skill acquisition process does not always produce linear changes in physical performance. Nonsystematic deviations are frequently observed at either the initial or the final tails of the skill acquisition functions and are attributed to changes in stages of learning and strategies (e.g., Newell \& Rosenbloom, 1981; Welford, 1987). Also, skilled athletes often describe instabilities in performance right before they achieve an improved level of performance.

This study is a starting point for further research. Like many naturalistic studies, it has limits because only snapshots of skill acquisition for a complex task learned over years of training were examined. It was not possible to equate skill levels within groups or years of experience. Also, replication would be difficult, because relatively few people have the skill to be considered experts. To advance our knowledge of higher order representations, complex motor skills should be studied within subjects in a longitudinal study. Researchers could then determine 
differences in the characteristics of representations and corresponding performance as the skill develops. This type of study would also begin to address the intriguing questions of whether the cognitive organizations of true experts really are different from those of other performers who do not reach the same level of performance and why some athletes never reach world-class status.

\section{REFERENCES}

AAUdiving HANDBOOK. (1985). Fort Lauderdale, FL: Swimming Hall of Fame.

Anderson, J. R. (1983). The architecture of cognition. Cambridge, MA: Harvard University Press.

Annett, J. (1995). Motor imagery: Perception or action? Neuropsychologia, 33, 1395-1417.

Colley, A. M. (1989). Learning motor skills: Integrating cognition and action. In A. M. Colley \& J. R. Beech (Eds.), Acquisition and performance of cognitive skills (pp. 167-189). New York: Wiley.

DECETY, J. (1991). Motor information may be important for updating the cognitive processes involved in mental imagery of movement. European Bulletin of Cognitive Psychology, 4, 415-426.

DeCETY, J. (1996). Do imagined and executed actions share the same neural substrate? Cognitive Brain Research, 3, 87-93.

DeCETy, J., \& Boisson, D. (1990). Effect of brain and spinal cord injuries on motor imagery. European Archives of Psychiatry \& Clinical Neurosciences, 240, 39-43.

Decety, J., \& IngVar, D. H. (1990). Brain structures participating in mental simulation of motor behavior: A neuropsychological interpretation. Acta Psychologica, 73, 13-34.

Decety, J., \& JeAnNerod, M. (1996). Fitts' law in mentally simulated movements. Behavioural Brain Research, 72, 127-134.

Decety, J., Jeannerod, M., \& Preblanc, C. (1989). The timing of mentally represented actions. Behavioural Brain Research, 34, 3542.

Decety, J., Kawashima, R., Gulyas, B., \& Roland, P. E. (1992). Preparation for reaching: A PET study of the participating structures in the human brain. NeuroReport, 3, 761-764.

Decety, J., \& Michel, F. (1989). Comparative analysis of actual and mental movement times in two graphic tasks. Brain \& Cognition, 11, 87-97.

Dominey, P., Decety, J., Broussolle, E., Сhazot, G., \& Jeannerod, M. (1995). Motor imagery of a lateralized sequential task is asymmetrically slowed in hemi-Parkinson's patients. Neuropsychologia, 33, 727-741.

Driskell, J., Copper, C., \& Moran, A. (1994). Does mental practice enhance performance? Journal of Applied Psychology, 79, 481-492.

Feltz, D. L., \& LANDERs, D. M. (1983). The effects of mental practice on motor skill learning and performance: A meta-analysis. Journal of Sport Psychology, 5, 25-57.

Feltz, D. L., Landers, D. M., \& Becker, B. J. (1988). A revised metaanalysis of the mental practice literature on motor skill learning. Washington, DC: National Academy Press.

Guilford, J. P., \& Zimmerman, W. (1947). Guilford-Zimmerman Aptitude Survey Test: Part VI. Spatial Visualization. Beverly Hills, CA: Sheridan Supply.

HaLe, B. D. (1982). The effects of internal and external imagery on muscular and ocular concomitants. Journal of Sport Psychology, 4, 374-387.

Harris, D. V., \& Robinson, W. J. (1986). The effects of skill level on EMG activity during internal and external imagery. Journal of Sport Psychology, 8, 105-111.

Holding, D. H. (ED.) (1989). Human skills (2nd ed.). Chichester, U.K.: Wiley.

IVRY, R. (1994). Representational issues in motor learning. In H. Heuer \& S. W. Keele (Eds.), Handbook of perception and action (Vol. 2, pp. 263-330). New York: Academic Press.
JACOBSON, E. (1932). Electrophysiology of mental activities. American Journal of Psychology, 44, 676-694.

JANNSON, L. H. (1983). Mental training: Thinking rehearsal and its use. In W. Maxwell (Ed.), Thinking: The expanding frontier (pp. 191197). Philadelphia: Franklin Press.

JEANNEROD, M. (1994). The representing brain: Neural correlates of motor intention and imagery. Brain \& Behavioral Sciences, 17, 187-245.

JeAnnerod, M. (1995). Mental imagery in the motor context. Neuropsychologia, 33, 1419-1432.

JEANNEROD, M. (1997). The cognitive neuroscience of action. Cambridge, MA: Blackwell.

JeANNEROD, M., \& FraK, V. (1999). Mental imaging of motor activity in humans. Current Opinion in Neurobiology, 9, 735-739.

Just, M. A., \& CARPENTER, P. A. (1985). Cognitive coordinate systems: Accounts of mental rotation and individual differences in spatial ability. Psychological Review, 92, 137-172.

Karmiloff-Smith, A. (1990). Constraints on representational change: Evidence from children's drawing. Cognition, 34, 57-83.

Karmiloff-Smith, A. (1992). Beyond modularity: A developmental perspective on cognitive science. Cambridge, MA: MIT Press, Bradford Books.

KeELE, S. W. (1968). Movement control in skilled motor performance. Psychological Bulletin, 70, 387-403.

KoHL, R. M., \& RoenKer, D. L. (1983). Mechanism involvement during skill imagery. Journal of Motor Behavior, 15, 179-190.

Kosslyn, S. M., Brunn, J., Cave, K. R. \& Wallach, R. W. (1984). Individual differences in mental imagery ability: A computational analysis. Cognition, 18, 195-243.

Kosslyn, S. M., \& Koenig, O. (1992). Wet mind: The new cognitive neuroscience. New York: Free Press.

Kosslyn, S. M., \& Schwartz, S. P. (1977). A simulation of visual imagery. Cognitive Science, 1, 265-298.

Landauer, T. K. (1962). Rate of implicit speech. Perceptual \& Motor Skills, 15, 646.

MACKAY, D. G. (1981). The problem of rehearsal or mental practice. Journal of Motor Behavior, 13, 274-285.

MACKAY, D. G. (1982). The problems of flexibility, fluency, and speed-accuracy trade-off in skilled behavior. Psychological Review, 89, 483-506.

MacKaY, D. G. (1989). The organization of perception and action. New York: Springer-Verlag.

McGuigan, F. J. (1970). Covert oral behavior during the silent performance of language tasks. Psychological Bulletin, 74, 309-326.

Minas, S. C. (1978). Mental practice of a complex perceptual motor skill. Journal of Human Movement Studies, 4, 102-109.

Newell, A., \& Rosenbloom, P. S. (1981). Mechanisms of skill acquisition and the law of practice. In J. R. Anderson (Ed.), Cognitive skills and their acquisition (pp. 1-55). Hillsdale, NJ: Erlbaum.

Ogden, J. A. (1985). Autotopagnosia: Occurrence in a patient without nominal aphasia and with an intact ability to point to parts of animals and objects. Brain, 108, 1009-1022.

PARSONS, L. M. (1987a). Imagined spatial transformation of ones' body. Journal of Experimental Psychology: General, 116, 172-191.

PARSONs, L. M. (1987b). Imagined spatial transformations of one's hands and feet. Cognitive Psychology, 19, 178-241.

PARSONS, L. M. (1994). Temporal and kinematic properties of motor behavior reflected in mentally simulated action. Journal of Experimental Psychology: Human Perception \& Performance, 20, 709-730.

ReED, C. L., \& Farah, M. J. (1995). Body schema: A study with normal participants. Journal of Experimental Psychology: Human Perception \& Performance, 21, 334-343.

REQUIN, J. (1991). Neural basis of movement representations. In J. Requin \& G. E. Stelmach (Eds.), Tutorials in motor neurosciences (pp. 333-345). Dordrecht: Kluwer.

Richardson, A. (1967). Mental practice: A review and discussion. Research Quarterly, 38, 95-107.

Rizzolatti, G., Fadiga, L., Gallese, V., \& Fogassi, L. (1996). Premotor cortex and the recognition of motor actions. Cognitive Brain Research, 3, 131-141. 
Roland, P. E., Larsen, B., Lassen, N. A., \& Skinhoj, E. (1980). Supplementary motor area and other cortical areas in organization of voluntary movements in man. Journal of Neurophysiology, 43, 118136.

Rosenbaum, D. A. (1991). Human motor control. New York: Academic Press.

Rosenbaum, D. A., Inhoff, A. W., \& Gordon, A. M. (1984). Choosing between movement sequences: A hierarchical control of rapid movement sequences. Journal of Experimental Psychology: Human Perception \& Performance, 9, 86-102.

Schmidt, R. A. (1975). A schema theory of discrete motor skill learning. Psychological Review, 32, 225-240.

SchmidT, R. A. (1988). Motor control and learning (2nd ed.). Champaign, IL: Human Kinetics.

SHAFFER, L. H. (1980). Analysing piano performance. In G. E. Stelmach \& J. Requin (Eds.), Tutorials in motor behavior (pp. 443-455). Amsterdam: North-Holland.

Shaffer, L. H. (1982). Rhythm and timing in skill. Psychological Review, 89, 109-122.

ShIFFrar, M., \& FreYd, J. J. (1993). Timing and apparent motion path choice with human body photographs. Psychological Science, 4, 379384.

Sirigu, A., Duhamel, J. R., Cohen, L., Pillon, B., Dubois, B., \& AgID, Y. (1996). The mental representation of hand movements after parietal cortex damage. Science, 273, 1564-1568.

Thurstone, L. L., \& Jeffreys, T. E. (1956). Flags: A test of spatial thinking. Chicago: Chicago Industry Service.

Ulich, E. (1967). Some experiments on the function of mental training in the acquisition of motor skills. Ergonomics, 10, 411-419.
Welford, A. T. (1987). On rates of improvement with practice. Journal of Motor Behavior, 19, 401-415.

\section{NOTES}

1. Separate group $\times$ performance type $\times$ waypoint ANOVAs were conducted for each dive, using mean waypoint data. Each analysis revealed a significant three-way interaction [front dive, $F(6,57)=3.23$, $M S_{\mathrm{e}}=0.075, p<.009$; front somersault, $F(6,57)=2.55, M S_{\mathrm{e}}=0.01, p<$ .03 ; back twister, $F(4,38)=3.11, M S_{\mathrm{e}}=0.04, p<.03$ ]

2. Data were analyzed using linear regressions with dummy variables and no constant. A dummy variable was created for group, Dummy group, where dummy ${ }_{1}=1$ iff group $=1$, otherwise dummy ${ }_{1}=0$. Dummy variables were also created in which group and dive had specific values: Dummy $_{\text {group,dive }}$, where dummy ${ }_{11}=1$ iff group $=1$, dummy $=1$, and 0 other wise. This analysis permitted performances to be compared across dives with an unbalanced design, as well as a test of heteroscedasticity to account for unequal variances in the cells. This was important because the measures are nonindependent and because variable error could be a function of dive difficulty, group, and other variables. Despite these precautions, only the results from standard analyses are reported, because they produced results similar to the analyses with controls for heteroscedasticity.

3 . Spatial ability groups were determined by ranking scores from highest to lowest. The top eight scores were assigned to the high spatial ability group ( 2 experts, 3 intermediates, 3 novices). The next seven scores were the intermediate spatial ability group ( 3 experts, 2 intermediates, 2 novices). The remaining seven scores were the low spatial ability group ( 2 experts, 3 intermediates, 2 novices). Spatial test scores were distributed across the range of possible scores.

\section{APPENDIX}

The front dive (Dive 101: forward dive, straight position) describes a simple trajectory motion. The diver stands on the anchored end of the diving board that lies above the pool deck. The dive begins when the diver initiates the walk to the opposite end of the board extending over the water. The diver subsequently jumps on the end of the board to propel himself or herself into the air. Once in the air, the diver's straight body pivots so that the feet rise, and the head lowers until the body is perpendicular to the surface of the pool water. The dive is completed as the diver enters the water headfirst.

The front somersault (Dive 10: forward dive, two-and-one-half somersault, pike position) adds a lateral body rotation to the simple trajectory of the front dive. The diver walks to the end of the board and jumps on the end of the board to propel himself or herself into the air. The diver subsequently bends at the waist and completes two-and-one-half revolutions around the body's lateral axis. When the revolutions are completed, the diver straightens his or her body so that it is perpendicular to the surface of the water and enters the water headfirst to complete the dive.

The back twister (Dive 5,231: back dive, one-and-one-half somersault, one-and-one-half twists, free position) requires both a lateral and a vertical body rotation. To initiate the dive, the diver stands at the pool end of the diving board and faces the pool deck. The diver propels himself or herself off the board by pressing down toward the board with his or her arms, bending his or her knees, and jumping into the air. The diver executes one-and-onehalf rotations around the body's vertical axis (the twist portion) while simultaneously executing one-and-one-half revolutions about the body's lateral axis (the somersaultportion). The dive is completed when the diver enters the water headfirst, with his or her body perpendicular to the water surface. 